\title{
Pegylated Recombinant Human Arginase 1 Induces Autophagy and Apoptosis via the ROS-Activated AKT/mTOR Pathway in Bladder Cancer Cells
}

\author{
Zhuyun Zhao $\mathbb{D}^{1}{ }^{1}$ Peng Zhang $\mathbb{D}^{2}{ }^{2}$ Wei Li $\mathbb{D}^{3}$, Dengchuan Wang $\mathbb{D}^{4},{ }^{4}$ Changneng Ke $\mathbb{D},{ }^{1}$ \\ Yueming Liu $₫$, , James Chung-Man Ho $₫{ }^{5},{ }^{1}$ Paul Ning-Man Cheng, ${ }^{6}$ and Shi Xu ${ }^{1}$ \\ ${ }^{1}$ Department of Burn and Plastic Surgery, Shenzhen Longhua District Central Hospital, Affiliated Central Hospital of Shenzhen \\ Longhua District, Guangdong Medical University, Shenzhen, Guangdong, China \\ ${ }^{2}$ Department of Pharmacy, Shenzhen Luohu People's Hospital, Shenzhen, Guangdong, China \\ ${ }^{3}$ Department of Urology, Shenzhen Longhua District Central Hospital, Affiliated Central Hospital of Shenzhen Longhua District, \\ Guangdong Medical University, Shenzhen, Guangdong, China \\ ${ }^{4}$ Office of Medical Ethics, Shenzhen Longhua District Central Hospital, Affiliated Central Hospital of Shenzhen Longhua District, \\ Guangdong Medical University, Shenzhen, Guangdong, China \\ ${ }^{5}$ Division of Respiratory Medicine, Department of Medicine, The University of Hong Kong, Queen Mary Hospital, Pokfulam, \\ Hong Kong SAR, China \\ ${ }^{6}$ Bio-Cancer Treatment International, 511-513, Bioinformatics Building, Hong Kong Science Park, Tai Po, Hong Kong SAR, China
}

Correspondence should be addressed to Shi Xu; xushi_cn@163.com

Received 14 January 2021; Revised 22 February 2021; Accepted 5 March 2021; Published 18 March 2021

Academic Editor: Jos L. Quiles

Copyright (C) 2021 Zhuyun Zhao et al. This is an open access article distributed under the Creative Commons Attribution License, which permits unrestricted use, distribution, and reproduction in any medium, provided the original work is properly cited.

Bladder cancer is one of the most commonly diagnosed cancers worldwide, especially in males. Current therapeutic interventions, including surgery, radiation therapy, chemotherapy, and immunotherapy, have not been able to improve the clinical outcome of bladder cancer patients with satisfaction. Recombinant human arginase (rhArg, BCT-100) is a novel agent with great anticancer effects on arginine-auxotrophic tumors. However, the effects of BCT-100 on bladder cancer remain unclear. In this study, the in vitro anticancer effects of BCT-100 were assessed using four bladder cancer cell lines (J82, SCaBER, T24, and 5637), while the in vivo effects were evaluated by establishing T24 nude mice xenograft models. Intracellular arginine level was observed to be sharply decreased followed by the onset of apoptotic events. Furthermore, BCT-100 was found to induce $\mathrm{H}_{2} \mathrm{O}_{2}$ production and mitochondrial membrane depolarization, leading to the release of mitochondrial cytochrome c and Smac to the cytosol. Treatment with BCT was observed to upregulate the expression of LC3B and Becllin-1, but downregulate the expression of p62 in a time-dependent manner. Autophagic flux was also observed upon BCT-100 treatment. Besides, the phosphorylation of the AKT/mTOR pathway was suppressed in a time-dependent fashion in BCT-100-treated T24 cells. While N-acetyl-L-cysteine was shown to alleviate BCT-100-induced apoptosis and autophagy, chloroquine, MK-2206, and rapamycin were found to potentiate BCT-100-triggered apoptosis. Finally, BCT-100 was demonstrated to induce autophagy and apoptosis via the ROS-mediated AKT/mTOR signaling pathway in bladder cancer cells.

\section{Introduction}

Bladder cancer is one of most prevalent diseases worldwide, especially in male. It was estimated that bladder cancer accounts for approximately $3.0 \%$ of all new cancer diagnoses and $2.1 \%$ of cancer deaths [1]. Urothelial cell carcinoma is the most commonly diagnosed bladder cancer subtype, followed by squamous cell carcinomas [2]. Similar to lung cancer, tobacco use is the leading cause of bladder cancer among other risk factors, which include arsenic exposure, chlorine exposure, and genetics [3-5]. Currently, used standard treatments for bladder cancer patients include surgery, 
radiation therapy, chemotherapy, and immunotherapy [6, 7]. Although chemotherapy based on the combined use of cisplatin and gemcitabine is currently applied as the first-line treatment strategy for bladder cancer, the emergence of immunotherapy has facilitated the improvement of the overall patient survival rate. However, due to renal insufficiency or cancer cachexia, nearly half of the patients have not been able to benefit from this treatment method $[6,8]$. Hence, there is an urgent requirement for the development of novel and safe therapeutic approaches that can improve the clinical outcomes of bladder cancer patients.

Arginine is a semiessential amino acid for human, which exerts multifaceted functions in cellular activities, including cell growth, metabolism, and survival [9]. Arginine is an essential precursor in the synthesis of protein, nitric oxide, polyamines, creatinine, and nucleotides [10]. An in-depth study on the various aspects of tumor metabolism has revealed that metabolic therapy may be a promising treatment option [11]. It has been demonstrated that certain cancer cells are incapable of synthesizing arginine independently, and they are reliant on extracellular arginine for growth $[12,13]$. Therefore, arginine deprivation may be a treatment intervention for arginine auxotrophic cancers, including hepatic cell carcinoma, prostate cancer, melanoma, small-cell lung cancer, and leukemia [12-16].

Theoretically, arginine can be metabolized by five enzymatic factors (i.e., nitric oxide synthase, glycine amidinotransferase, arginine decarboxylase, arginine deiminase (ADI), and arginase) to induce arginine depletion. Due to a number of factors, such as drug efficacy, immunogenicity, drug half-time, and potential byproduct generation, only PEGylated ADI and arginase have been employed for the treatment of arginine auxotrophic cancers [17]. An increasing number of studies have shown that two important enzymes in the urea cycle, argininosuccinate synthase (ASS1) and ornithine transcarbamylase (OTC), can greatly affect the efficacy of arginine depletors $[13,18,19]$.

In this study, we used BCT-100, which is a classic recombinant human arginase. Consistent with endogenous arginase, BCT-100 catalyzes the conversion of arginine to ornithine and urea, leading to arginine depletion [19]. It has been demonstrated that BCT-100 exhibit significant anticancer effects towards several arginine auxotrophic cancers, and completed clinical trials of BCT-100 on hepatocellular carcinoma (HCC) result in promising outcomes $[12,13$, 20]. However, very few studies have investigated the role of BCT-100 on bladder cancer. Here, we explored the underlying mechanisms of arginine deprivation in bladder cancer using BCT-100, which may be used as a potential therapeutic approach.

\section{Materials and Methods}

2.1. Cell Lines and Cell Culture. A panel of four bladder cell lines (J82, SCaBER, T24, and 5637), one human bladder cell (SV-HUC-1), and one non-small-cell lung cancer cell line (A549) were purchased from the American Type Culture Collection (Manassas, VA, USA). All cell lines were maintained in RPMI-1640 medium (Gibco, Life Technologies,
Carlsbad, California, USA) supplemented with $10 \%$ fetal bovine serum (FBS) (Gibco, USA) in a humidified atmosphere containing $5 \% \mathrm{CO}_{2}$ at $37^{\circ} \mathrm{C}$.

2.2. Reagents. BCT-100 was kindly provided by Bio-Cancer Treatment International Limited, Hong Kong. N-Acetyl-Lcysteine (NAC, Cat\# A7250) and chloroquine (CQ, Cat\# C6628) were purchased from Sigma. MK-2206 (Cat\# SF2712-5 mg) and rapamycin (Cat\# S1842-25 mg) were bought from Beyotime Biotechnology.

2.3. Western Blot Analysis. Treated cells were harvested, washed, and resuspended in NP-40 lysis buffer (Beyotime Biotechnology, Jiangsu, China) supplemented with protease inhibitor (1 mM phenylmethylsulphonyl fluoride) for $1 \mathrm{~h}$. Total protein from xenograft samples was extracted using tissue protein extraction reagent kit (Thermo, Cat\# 78510). Supernatants were collected after centrifugation (13000 rpm, $\left.4^{\circ} \mathrm{C}, 30 \mathrm{~min}\right)$. For mitochondrial and cytosolic protein extraction, cell mitochondria isolation kit (Beyotime Biotechnology, Cat\# C3601) was used. Protein concentration was measured using Bradford Protein Assay Kit (Bio-Rad, Berkeley, CA, USA). Extracted protein (30-60 $\mu \mathrm{g})$ was separated using $8-15 \%$ sodium dodecyl sulfate polyacrylamide gel electrophoresis (SDS-PAGE) and subsequently electroblotted onto polyvinylidene fluoride (PVDF) membranes (Millipore, MA, USA). The membranes were blocked for $1 \mathrm{~h}$ at room temperature in 5\% nonfat dry milk (dissolved in PBST solution) before being incubated overnight at $4^{\circ} \mathrm{C}$ with monoclonal or polyclonal primary antibodies (Table 1), followed by incubation with horseradish peroxidase- (HRP-) conjugated secondary antibodies (Cell Signaling Technology, MA, USA) for $1 \mathrm{~h}$ at room temperature. Signal detection was conducted using enhanced chemiluminescence (ECL) kit (GE Healthcare). Quantification was performed using GelQuantNET software (Biochem Lab Solutions, CA, USA).

2.4. Cell Viability Assay. Cells were seeded in a 96-well plate at a density of approximately $10^{4}$ cells per well. After drug exposure, cells were incubated with $10 \mu \mathrm{l}$ of 3-(4,5-dimethylthiazol-2-yl)-2,5-diphenyltetrazolium bromide (MTT; Sigma-Aldrich) at $0.5 \mathrm{mg} / \mathrm{ml}$ for $3 \mathrm{~h}$, followed by $100 \mu \mathrm{l}$ triple lysis solution (10\% sodium dodecyl sulfate, $5 \%$ isobutanol, and $0.012 \mathrm{~mol} / \mathrm{l} \mathrm{HCl}$ in water) for $2 \mathrm{~h}$. Optical density was measured at $570 \mathrm{~nm}$ using FLUOstar OPTIMA microplate reader (BMG Labtech GmbH, Ortenberg, Germany).

2.5. Arginine Concentration Detection. The levels of arginine in cells, serum, and tumor were measured using L-arginine ELISA kit (Abcam, Cat\# ab241028, MA, USA) according to the manufacturer's instructions. In brief, derivatized samples and standards were incubated with L-arginine antibody overnight at $4^{\circ} \mathrm{C}$ before being incubated with peroxidase conjugate for $1 \mathrm{~h}$ at room temperature and with tetramethylbenzidine substrate for $10 \mathrm{~min}$ in the dark. The samples were washed with wash buffer in between each step. Stop solution was added to halt the reaction. Absorbance values at $450 \mathrm{~nm}$ (using $620 \mathrm{~nm}$ as the reference) were 
TABLE 1: List of primary antibodies used in this study.

\begin{tabular}{|c|c|c|c|c|c|}
\hline Name & Species & Manufacturer & Cat\# & Molecular weight $(\mathrm{kDa})$ & Dilution factor \\
\hline ASS1 & Rabbit & Santa Cruz Biotechnology & SC-99178 & 47 & $1: 500$ \\
\hline C-PARP & Rabbit & Santa Cruz Biotechnology & SC-7150 & 89 & $1: 1000$ \\
\hline PEG & Rabbit & RevMAb Biosciences & $31-1008-00$ & 200 & $1: 1000$ \\
\hline OTC & Rabbit & Santa Cruz Biotechnology & SC-102051 & 40 & $1: 200$ \\
\hline m-TOR & Rabbit & Cell Signaling Technology & 2972 & 289 & $1: 1000$ \\
\hline p-mTOR & Rabbit & Cell Signaling Technology & 5526 & 289 & $1: 1000$ \\
\hline Survivin & Rabbit & Cell Signaling Technology & 2808 & 16 & $1: 1000$ \\
\hline Smac & Mouse & Cell Signaling Technology & 2954 & 21 & $1: 1000$ \\
\hline Cyt-c & Rabbit & Cell Signaling Technology & 4280 & 14 & $1: 1000$ \\
\hline COX IV & Mouse & Cell Signaling Technology & 11967 & 17 & $1: 1000$ \\
\hline AKT & Rabbit & Cell Signaling Technology & 9272 & 60 & $1: 1000$ \\
\hline p-AKT & Rabbit & Cell Signaling Technology & 4060 & 60 & $1: 1000$ \\
\hline p62 & Mouse & Cell Signaling Technology & 88588 & 62 & $1: 1000$ \\
\hline LC3B & Rabbit & Cell Signaling Technology & 3868 & 14 & $1: 1000$ \\
\hline Beclin-1 & Rabbit & Cell Signaling Technology & 3495 & 60 & $1: 1000$ \\
\hline Caspase 3 & Rabbit & Cell Signaling Technology & 9662 & 35 & $1: 1000$ \\
\hline$\beta$-Actin & Mouse & Sigma-Aldrich & A5441 & 42 & $1: 5000$ \\
\hline
\end{tabular}

measured using FLUOstar OPTIMA microplate reader (BMG Labtech GmbH, Ortenberg, Germany).

2.6. Short Hairpin RNA (shRNA) Transfection. Silencing of ASS1 (shRNA) was conducted using a lentiviral particles kit bought from Santa Cruz (Cat\# sc-45810-v). In brief, cells were pretreated with polybrene prior to incubation with lentiviral particles for $24 \mathrm{~h}$. Following the removal of unbound viral particles, cells were further incubated with fresh complete medium for $48 \mathrm{~h}$. Stable ASS1-silenced cell line was selected using puromycin dihydrochloride. The expression of ASS1 protein was evaluated by Western blot after the cells were confirmed to be viable by MTT assay.

2.7. Autophagic Flux Analysis. Autophagic flux in T24 cells was detected using mRFP-GFP-LC3 adenovirus (Hanbio, China). Cells were seeded in a 6-well plate at a density of 1 $\times 10^{5}$ cells/dish before being incubated with mRFP-GFPLC3 adenovirus with or without BCT-100 $(20 \mathrm{mU} / \mathrm{ml})$ for $24 \mathrm{~h}$. Autophagic flux was observed under an inverted fluorescent microscope (Zeiss, Germany). Yellow and red puncta indicate autophagosomes and autolysosome, respectively.

2.8. Flow Cytometry of Annexin VIPI Staining. Apoptosis was determined by flow cytometry using FITC-conjugated Annexin V/PI kit (Beyotime Biotechnology, Jiangsu, China). Briefly, cells were harvested, washed, and resuspended in binding buffer at $5 \times 10^{6} \mathrm{ml}^{-1}$. $5 \mu \mathrm{l}$ of FITC-conjugated Annexin V solution and $10 \mu \mathrm{l}$ of PE-conjugated PI solution were added to $100 \mu \mathrm{l}$ of cell suspension. After incubation with Annexin V/PI for $15 \mathrm{~min}$ at room temperature, samples were measured using BD FACSAria II analyzer with FL2/FL4 channels (BD, New Jersey, USA). Every sample counted 10000 events for quantification.
2.9. Reactive Oxygen Species (ROS) Measurement. The presence of hydrogen peroxide $\left(\mathrm{H}_{2} \mathrm{O}_{2}\right)$ was tested using $2^{\prime}, 7^{\prime}$ -dichlorodihydro-fluorescein diacetate (H2DCFDA; Beyotime Biotechnology, Jiangsu, China). Briefly, treated cells were harvested, washed, and incubated with H2DCFDA $(1 \mu \mathrm{M})$ in medium without FBS for $30 \mathrm{~min}$ at $37^{\circ} \mathrm{C}$, followed by washing twice before being analyzed by flow cytometry. The presence of reduced glutathione (GSH) was measured by 5 -chloro-methylfluorescein diacetate (CMFDA; Invitrogen, OR, USA). Treated cells were collected, washed, and incubated with CMFDA $(5 \mu \mathrm{M})$ for $30 \mathrm{~min}$ at $37^{\circ} \mathrm{C}$ in FBSfree medium, following which the medium was changed to complete medium and the cells were further incubated for $30 \mathrm{~min}$ prior to flow cytometry analysis.

2.10. Detection of Mitochondrial Membrane Depolarization $(M M D)$. Mitochondrial membrane potential $(\Delta \Psi \mathrm{m})$ was

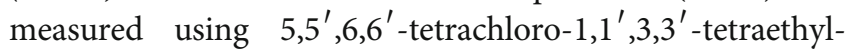
benzimidazolylcarbocyanine iodide (JC-1; Beyotime Biotechnology, Jiangsu, China). Briefly, treated cells were incubated in FBS-free medium with JC- $1(5 \mu \mathrm{g} / \mathrm{ml})$ at $37^{\circ} \mathrm{C}$ for $15 \mathrm{~min}$ in the dark before being washed with PBS. JC-1 fluorescent signal was determined by flow cytometry in FL1/FL2 channels.

2.11. Tumor Suppression Effect of BCT-100 Using Xenograft Models. Xenograft models were established by subcutaneous injection of $10^{7}$ cells into nude mice (4-5 weeks, 10-14 g, female, BALB/cAnN-nu, Laboratory Animal Centre of Shenzhen University, Guangdong, China). When the tumor reached the mean group size of $40-60 \mathrm{~mm}^{3}$, mice were randomly divided into three groups. PBS and BCT-100 (20 and $60 \mathrm{mg} / \mathrm{kg}$ ) were administered intraperitoneally twice a week. Tumor size was calculated according to the formula: $($ size $=$ length $\times$ width $\times$ depth $/ 2) \quad[21] . \quad$ Relative tumor 
volume (RTV) was calculated by the formula: RTV $=V_{n} / V_{0}$, where $V_{n}$ is tumor size on day $n$ and $V_{0}$ is tumor size on the first day of treatment. Mice were euthanized using sodium pentobarbital $(180 \mathrm{mg} / \mathrm{kg})$ when the tumor volume reached $600 \mathrm{~mm}^{3}$, which was regarded as a humane endpoint. The study protocol was approved by the laboratory animal ethical committee of Guangdong Medical University.

2.12. Terminal Deoxynucleotidyl Transferase-dUTP Nick End Labeling (TUNEL) Assay. TUNEL assay was conducted using Click-iT Plus TUNEL Assay kit (Beyotime Biotechnology, Jiangsu, China) according to the standard protocol provided by the manufacturer. Cell crawling, fixation, and permeabilization were required before TUNEL assay. Cells were immersed in terminal deoxynucleotidyl transferase (TdT) reaction buffer before being incubated in fresh $\mathrm{TdT}$ reaction buffer containing EdUTP, TdT, and TdT enzymes. Samples were incubated with TUNEL reaction cocktails followed by 4',6-diamidino-2-phenylindole (DAPI, Life Technologies, OR, USA) staining. Images were captured using a Zeiss fluorescence microscope.

2.13. Immunofluorescence. Fixed and permeabilized cells or tumor sections were blocked with $3 \%$ bovine serum albumin (BSA) for $1 \mathrm{~h}$ at room temperature, followed by incubation with ki67 antibody overnight at $4^{\circ} \mathrm{C}$. After washing with PBST for $30 \mathrm{~min}$, Alexa Fluor anti-rabbit (Life Technologies, OR, USA) antibody was applied for $1 \mathrm{~h}$ in the dark. Slides were mounted with ProLong Gold antifade reagent and DAPI. Images were obtained using a Zeiss fluorescence microscope.

2.14. Statistical Analysis. All data were obtained from at least three independent experiments and are shown as the mean \pm standard deviation (S.D.). Statistical analysis was performed using Student's two-tailed $t$-test by Prism 5 (GraphPad Software, La Jolla, Southern California, USA).

\section{Results}

3.1. Sensitivity of Bladder Cancer Cells to BCT-100 Correlated with the Expression of ASS1 and OTC. Firstly, the cell viability of four bladder tumor cell lines, including three transitional cell carcinomas (J82, T24, and 5637) and one squamous cell carcinoma (SCaBER), one human bladder cell line (SVHUC-1), and one lung cancer cell line (A549, positive control) treated with increasing concentrations of BCT-100 for 3 days, was evaluated by MTT assay. As shown in Figure 1(a), T24 and SCaBER cells had the highest $\left(\mathrm{IC}_{50}=20 \mathrm{mU} / \mathrm{ml}\right)$ and the lowest $\left(\mathrm{IC}_{50}=200 \mathrm{mU} / \mathrm{ml}\right)$ level of sensitivity to BCT-100 treatment, respectively. Next, the basal protein expression of ASS1 and OTC, two of the most important enzymes for arginine biosynthesis in the urea cycle, was evaluated by Western blot. ASS1 expression was relatively high in A549 and 5637 cells, whereas OTC was highly expressed in SCaBER and A549 cells (Figure 1(b)).

In order to explore the role of ASS1 upon BCT-100 exposure, ASS1 was knocked down in 5637 cells using lentiviral shRNA vector. Our results showed that knockdown of ASS1 increased the sensitivity of 5637 cells to BCT-100
(Figure 1(c)), and that the expression of cleaved poly ADPribose polymerase (C-PARP) was upregulated in ASS1silenced cells after BCT-100 exposure for 3 days (Figure 1(d)).

Since T24 cells were shown to be relatively sensitive to BCT-100 treatment, this cell line was selected as the model cell line in the following experiments to study the underlying mechanism of anticancer effect of BCT-100 in bladder cancer.

3.2. BCT-100 Induced Cytotoxicity and Lowered the Level of Arginine in Bladder Cancer Cells. BCT-100, a derivative of recombinant human arginase conjugated to polyethylene glycol (PEG), is used to indicate the accumulation and localization of arginase in vitro and in vivo. While PEG was detected in T24 cells upon BCT-100 exposure in a dosedependent manner (Figure 2(a)), intracellular arginine concentration was significantly decreased in a dose-dependent manner (Figure 2(b)). Meanwhile, cleaved PARP (C-PARP) was upregulated, and Caspase 3 and Survivin were downregulated, which was an indication of cytotoxicity induced by BCT-100 treatment (Figure 2(c)). Annexin V/PI staining (Figure 2(d)) and TUNEL assay (Figure 2(e)) showed an increase in the number of apoptotic cells. The apoptotic rate of T24 cells treated with BCT-100 at $20 \mathrm{mU} / \mathrm{ml}$ and $40 \mathrm{mU} / \mathrm{ml}$ was increased sharply to $32.0 \pm 6.3 \%$ and $48.0 \pm$ $4.1 \%$, respectively, compared to that of untreated cells $(4.0 \pm 2.4 \%)$.

3.3. Effect of BCT-100 on ROS Production, Mitochondrial Membrane Depolarization, and Mitochondria-Dependent Apoptosis. Intracellular hydrogen peroxide $\left(\mathrm{H}_{2} \mathrm{O}_{2}\right)$ was detected using fluorescence probe DCFH-DA. As shown in Figure 3(a), the production of $\mathrm{H}_{2} \mathrm{O}_{2}$ was significantly induced upon BCT-100 exposure for $24 \mathrm{~h}$. The relative percentage of DCF fluorescence intensity was boosted from 2.5 $\pm 1.9 \%$ (control) in untreated cells to $16.1 \pm 8.3 \%$ $(10 \mathrm{mU} / \mathrm{ml}), \quad 29.1 \pm 8.1 \% \quad(20 \mathrm{mU} / \mathrm{ml}), \quad$ and $\quad 41.0 \pm 6.3 \%$ $(40 \mathrm{mU} / \mathrm{ml})$ in BCT-100-treated cells. The level of reduced glutathione (GSH), as indicated by CMFDA staining, was observed to be decreased in a dose-dependent manner upon BCT-100 treatment for $24 \mathrm{~h}$ (Figure S1). Since mitochondria are essential for the production of ROS, mitochondrial membrane potential was measured using JC-1 staining. As shown in Figure 3(b), mitochondrial membrane depolarization occurred in T24 cells upon BCT-100 exposure for 3 days. The percentage of JC-1-positive cells in the control, $10 \mathrm{mU} / \mathrm{ml}, 20 \mathrm{mU} / \mathrm{ml}$, and $40 \mathrm{mU} / \mathrm{ml}$ group was $4.1 \pm 1.6 \%, 10.3 \pm 2.1 \%, 18.5 \pm 5.0 \%$, and $29.3 \pm 1.8 \%$, respectively. Next, the subcellular localization of Smac and cytochrome c (Cyt-c), both playing a crucial role in mitochondrial-dependent apoptosis, was investigated. As shown in Figures 3(c) and 3(d), while the levels of mitochondrial Smac and Cyt-c were decreased in cells treated with BCT-100 in a dose-dependent fashion, the levels of cytosolic Smac and Cyt-c were upregulated.

3.4. BCT-100 Induced Autophagy and Activated the AKT/mTOR Pathway. To evaluate the extent of autophagy 


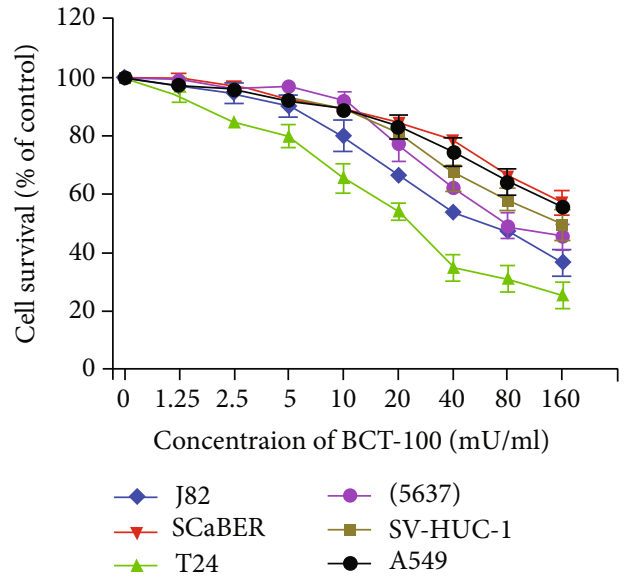

(a)

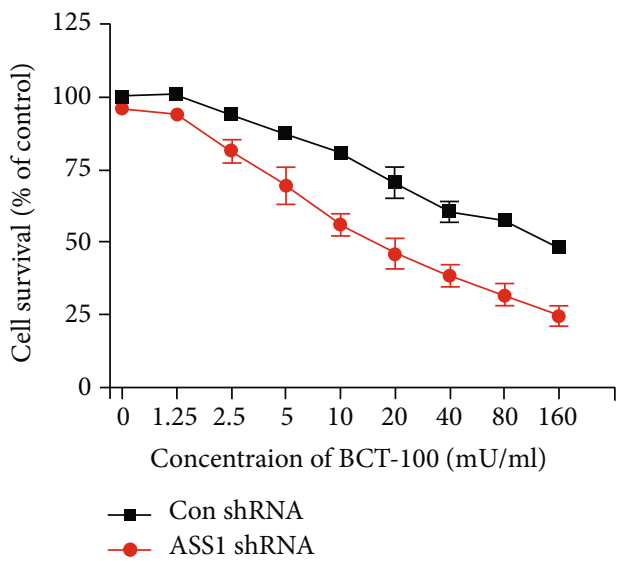

(c)
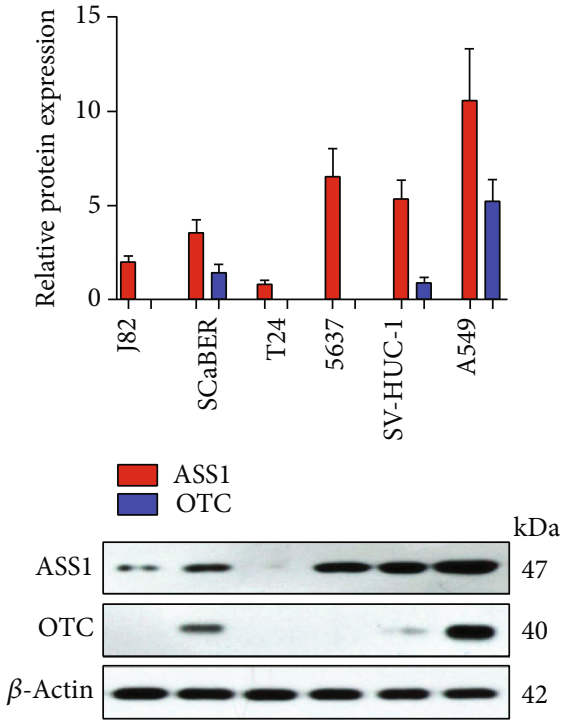

(b)

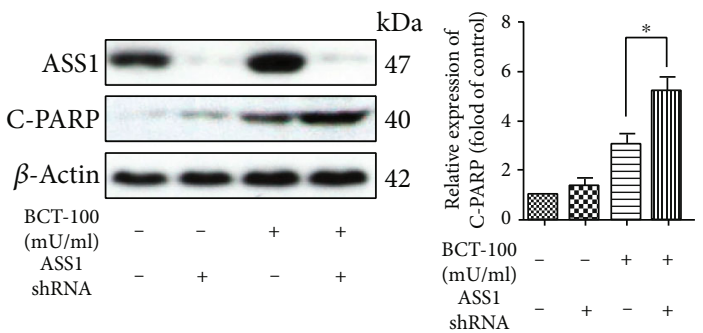

(d)

FIGURE 1: Sensitivity of bladder cancer cells to BCT-100 correlated with the expression of ASS1 and OTC. (a) Cell growth inhibition evaluated by MTT assay in bladder cancer cell lines (J82, SCaBER, T24, and 5637), human bladder cell line (SV-HUC-1), and A549 cells treated with BCT-100 for $72 \mathrm{~h}$. (b) Basal expression levels of ASS1 and OTC in bladder cancer cells evaluated by Western blot. (c) Cell survival rate of ASS1-silenced 5637 cells $(72 \mathrm{~h})$ evaluated by MTT assay. (d) Expression of C-PARP and ASS1 in ASS1-silenced 5637 cells treated with BCT-100 for $72 \mathrm{~h}$. $\beta$-Actin was used as a loading control.

induced by BCT-100, T24 cells were transfected with mRFPGFP-LC3 adenovirus. As displayed in Figure 4(a), the number of red and yellow (merged) puncta was remarkably increased in BCT-100-treated cells. Additionally, upon BCT-100 stimulation, the expression levels of autophagyrelated biomarkers, Beclin-1 and LC3B, were upregulated in a time-dependent manner, whereas the expression level of p62 was downregulated (Figure 4(b)). Since the AKTmTOR pathway exerts important roles in cell proliferation and autophagy, the phosphorylation levels of AKT and mTOR in BCT-100-treated cells were evaluated. As shown in Figure 4(c), the phosphorylation of both AKT (Ser473) and mTOR (Ser2448) was inhibited in a time-dependent fashion.

3.5. BCT-100-Induced ROS Initiated Cellular Apoptosis and Autophagy in Bladder Cancer Cells. Our results above indi- cated that BCT-100 exposure in cells induced the production of ROS, which is a key regulator of cellular apoptosis and autophagy. To investigate the role of ROS in BCT-100mediated apoptosis and autophagy, we employed NAC, an effective ROS scavenger in our study. As shown in Figure 5(a), cellular ROS level was significantly decreased in cells treated with NAC and BCT-100 compared to that in cells treated with BCT-100 alone $(21.5 \pm 3.3 \%$ vs. 44.2 $\pm 12.4 \%)$. Meanwhile, the apoptotic rate of cells treated with NAC and BCT-100, compared to those treated with BCT-100 alone, was $14.1 \pm 5.9 \%$ and $39.6 \pm 7.4 \%$, respectively (Figure 5(b)). Moreover, NAC inhibited the expression of C-PARP as well as LC3B, but promoted the expression of p62 in combination arm (Figure 5(c)). Next, to clarify the role of autophagy in cells treated with BCT-100, $\mathrm{CQ}$, an autophagy inhibitor was recruited. As shown in Figure 5(d), cellular apoptosis was significantly induced upon 


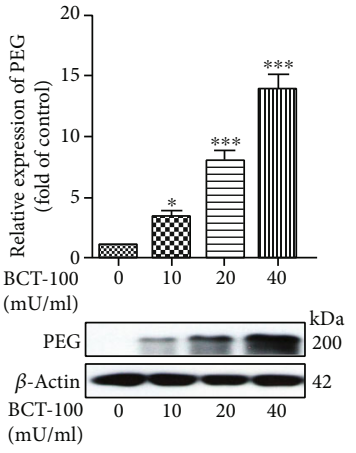

(a)

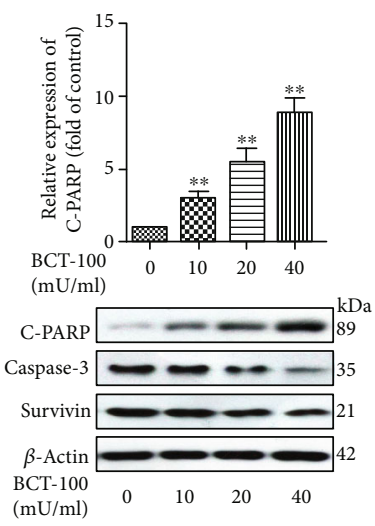

(c)
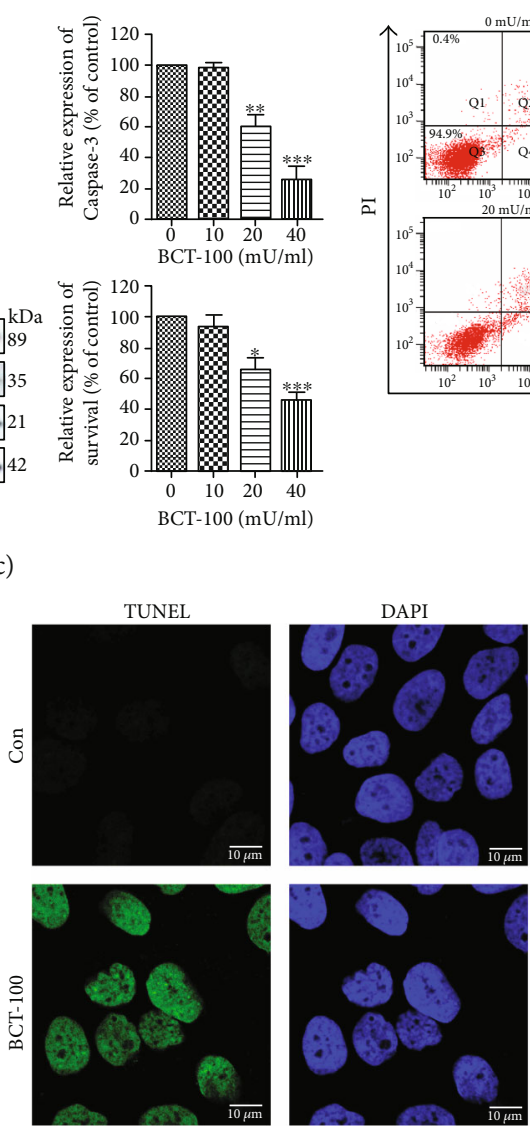

(e)

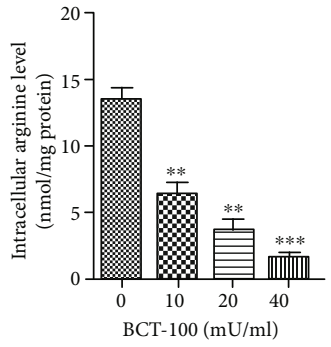

(b)
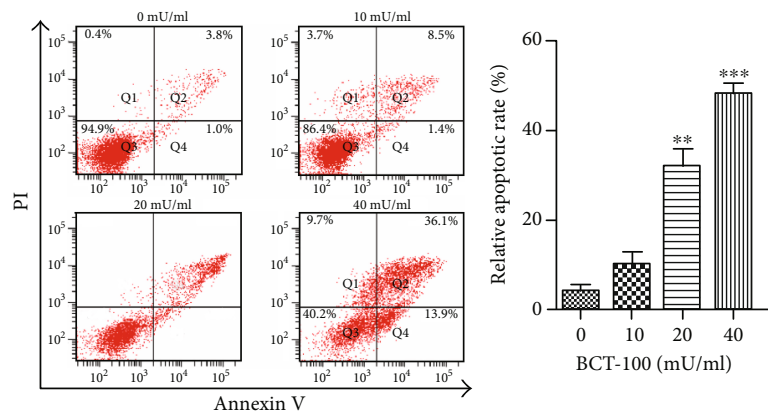

(d)
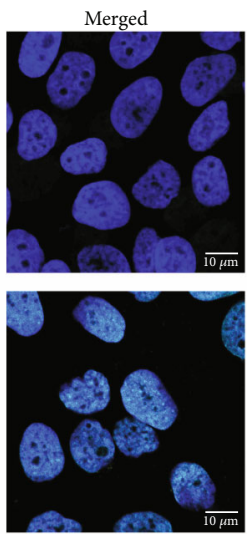

$\overline{10 \mu \pi}$

FIgure 2: BCT-100 induced cytotoxicity and lowered the level of arginine in bladder cancer cells. (a, c) Intracellular PEG, Caspase 3, Survivin, and C-PARP evaluated by Western blot in T24 cells exposed to BTC-100 $(20 \mathrm{mU} / \mathrm{ml})$ for $72 \mathrm{~h}$. (b) Intracellular arginine levels following BCT100 treatment for $72 \mathrm{~h}$. (d) Apoptotic rates determined by Annexin V and PI staining analyzed by flow cytometry in cells treated with BCT$100(20 \mathrm{mU} / \mathrm{ml})$ for $72 \mathrm{~h}$. (e) TUNEL assay indicating the presence of apoptotic cells exposed to BCT-100 (20 mU/ml) for $72 \mathrm{~h}$. Data are represented as the mean \pm S.D. of three independent experiments. ${ }^{*} p<0.05,{ }^{* *} p<0.01$, and ${ }^{* * *} p<0.001$, as evaluated by Student's $t$-test.

treatment with CQ and BCT-100, since the expression levels of C-PARP and Survivin were observed to be upregulated and downregulated, respectively, in the combination group. Consistently, cells exposed to CQ and BCT-100 showed increased accumulation of autolysosome (red puncta) and autophagosome (yellow puncta; Figure 5(e)). Furthermore, specific AKT and mTOR inhibitors (MK-2206 and rapamycin) were found to potentiate BCT-100-induced cytotoxicity, as indicated by the increased C-PARP expression coupled with decreased Survivin expression in cells exposed to CQ and BCT-100 (Figures 5(f) and 5(g)).

3.6. BCT-100 Suppressed Tumor Growth in T24 Xenograft Models. The tumor growth rate was observed to be significantly inhibited in mice receiving BCT-100 $(60 \mathrm{mg} / \mathrm{kg})$ treatment compared to that in mock-treated mice (Figure 6(a)). 

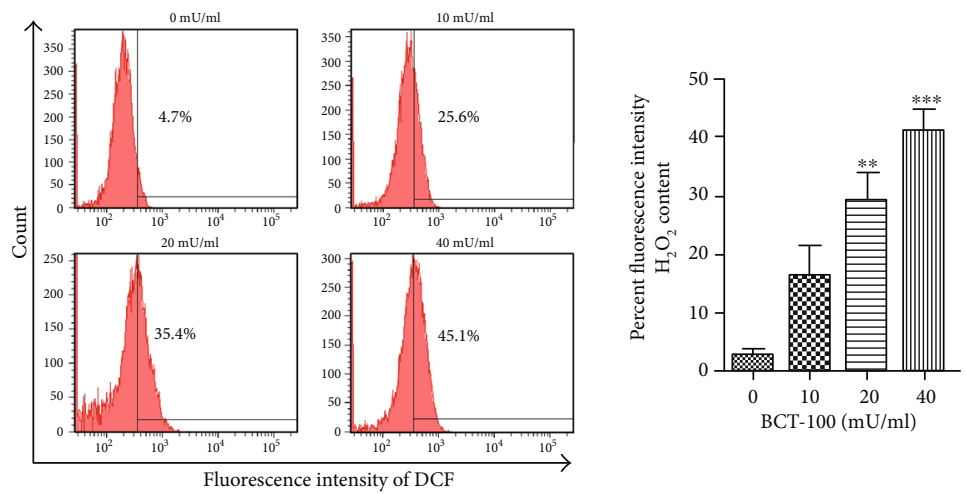

(a)
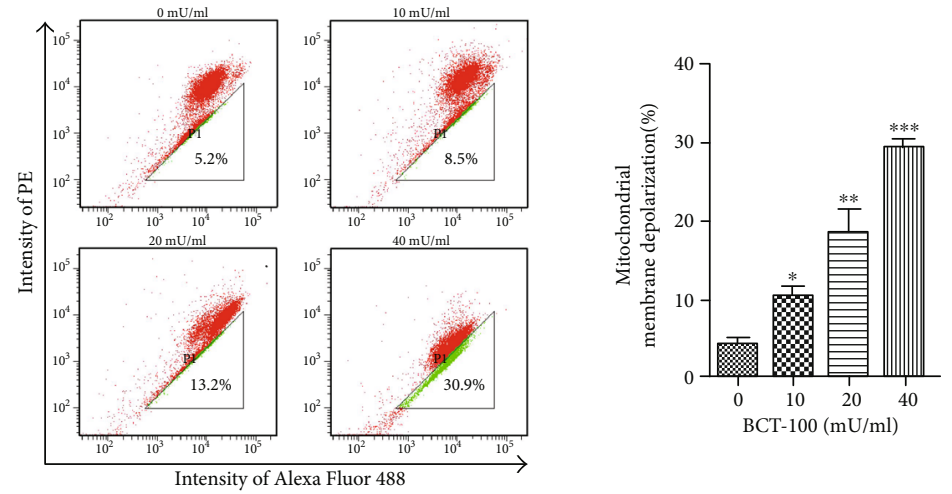

(b)
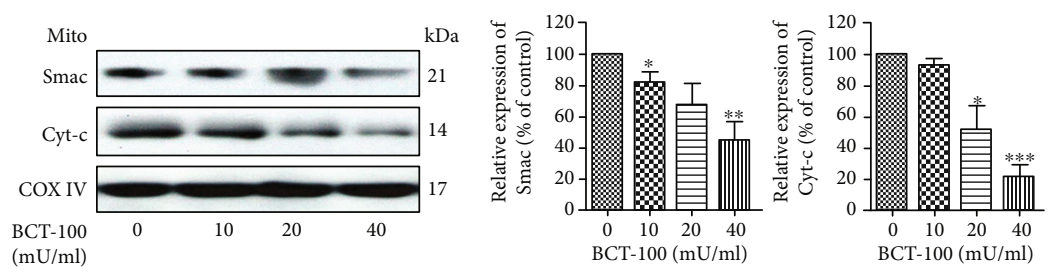

(c)
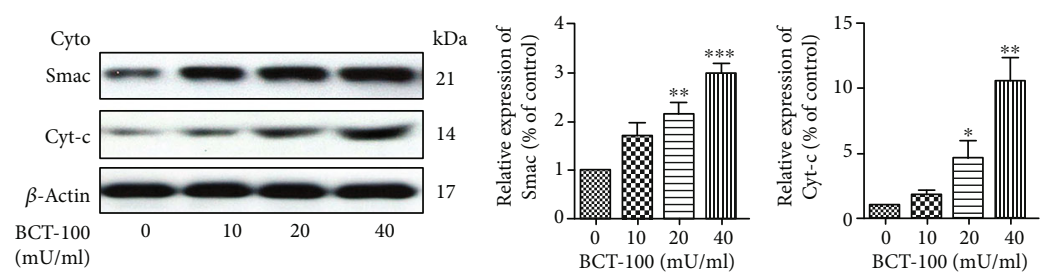

(d)

Figure 3: Apoptosis induced by BCT-100 was mitochondrial-dependent and accompanied by ROS production. (a) Elevation of $\mathrm{H}_{2} \mathrm{O}_{2}$ after BCT-100 treatment $(20 \mathrm{mU} / \mathrm{ml})$ for $24 \mathrm{~h}$ analyzed by flow cytometry. (b) Mitochondrial membrane depolarization shown by JC-1 staining in T24 cells upon BCT-100 (20 mU/ml) exposure for $24 \mathrm{~h}$. (c, d) Subcellular localization of Cyt-c and Smac assessed by Western blot in cells treated with BCT-100 $(20 \mathrm{mU} / \mathrm{ml})$ for $72 \mathrm{~h}$. COX IV and $\beta$-actin were used as loading controls in mitochondrial and cytosolic fraction, respectively. Data are represented as the mean \pm S.D. of three independent experiments. ${ }^{*} p<0.05,{ }^{* *} p<0.01$, and ${ }^{* * *} p<0.001$, as evaluated by Student's $t$-test.

In addition, the median survival of mice was prolonged following BCT-100 treatment from 25.0 days (mock-treated control group) to 26.5 days $(20 \mathrm{mg} / \mathrm{kg}$ treatment group) and to 31.5 days $(60 \mathrm{mg} / \mathrm{kg}$ treatment group; Figure $6(\mathrm{~b}))$. Next, the accumulation of PEG, LC3B, and apoptotic biomarkers in BCT-100-treated mice was investigated. In line with our in vitro results earlier, the levels of accumulated PEG, LC3B, and C-PARP were observed to be increased sharply, whereas the level of Survivin was found to be decreased (Figure 6(c)). Meanwhile, the concentrations of serum arginine in mice from the control group, $20 \mathrm{mg} / \mathrm{kg}$ treatment group, and $60 \mathrm{mg} / \mathrm{kg}$ treatment group were found 

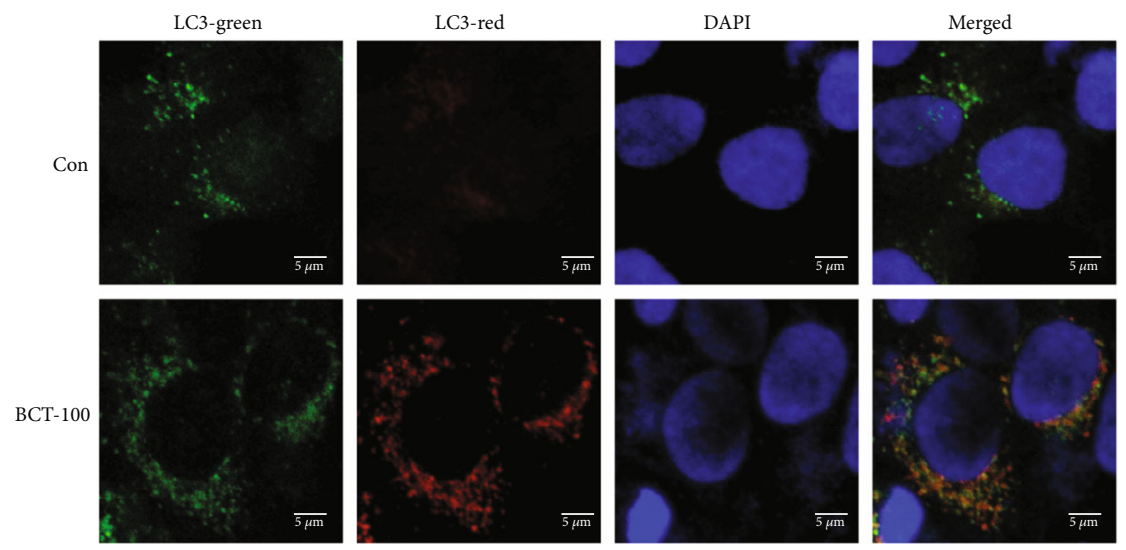

(a)
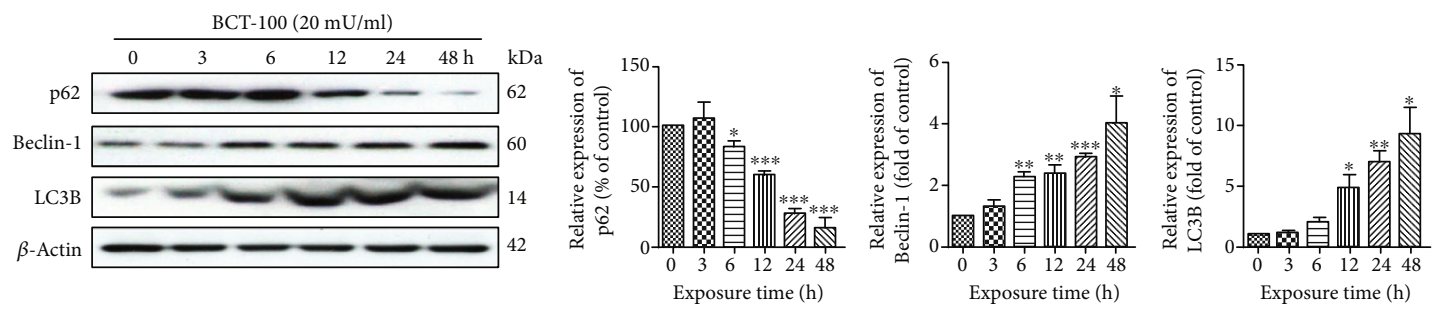

(b)
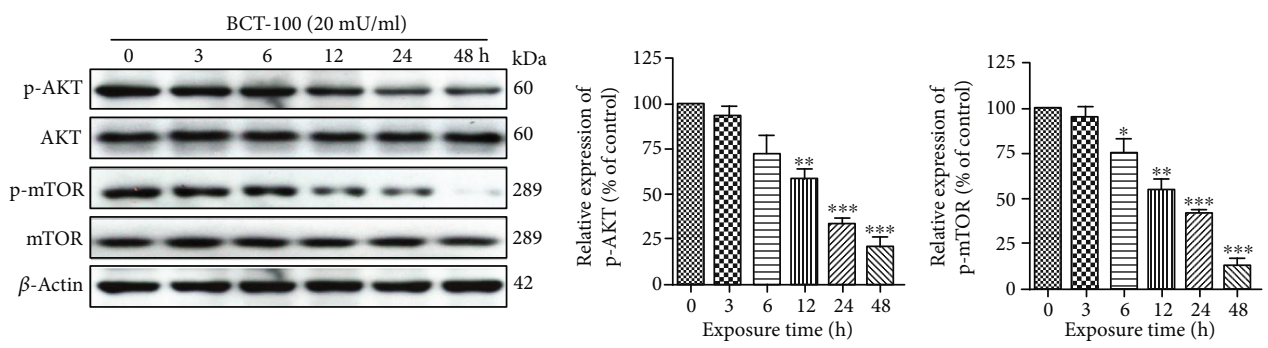

(c)

Figure 4: Autophagy induction and AKT/mTOR pathway activation after BCT-100 exposure. (a) T24 cells were transfected with the mRFPGFP-LC3 adenovirus for $24 \mathrm{~h}$ and treated with BCT-100 $(20 \mathrm{mU} / \mathrm{ml})$ for another $24 \mathrm{~h}$. Representative pictures of fluorescent LC3 puncta were captured by an inverted fluorescent microscope. (b, c) BCT-100-treated T24 cells were harvested at different time points. LC3B, p62, Beclin-1, p-AKT, AKT, p-mTOR, mTOR, and $\beta$-actin were tested by Western blot. All data are presented as the mean \pm S.D. of three independent experiments. ${ }^{*} p<0.05,{ }^{* *} p<0.01$, and ${ }^{* * *} p<0.001$, as evaluated by Student's $t$-test.

to be $72.7 \pm 14.4 \mu \mathrm{M}, 11.8 \pm 3.5 \mu \mathrm{M}$, and $5.6 \pm 2.6 \mu \mathrm{M}$, respectively (Figure $6(\mathrm{~d})$ ). Similarly, the levels of intratumoral arginine in mice were observed to be declined dramatically from $10.8 \pm 1.1 \mu \mathrm{M}$ (control group) to $4.7 \pm 0.5 \mu \mathrm{M}$ (20 mg/kg treatment group) and to $1.6 \pm 0.3 \mu \mathrm{M}(60 \mathrm{mg} / \mathrm{kg}$ treatment group; Figure 6(e)). Finally, the fluorescence intensity of Ki67, a biomarker associated with cell proliferation, was found to be decreased in a dose-dependent manner in mice treated with BCT-100 (Figure 6(f)).

\section{Discussion}

In this study, PEGylated recombinant human arginase 1 (BCT-100) was shown to sharply deplete the levels of intracellular and intratumoral arginine and display an antiproliferative effect on tumor growth in bladder cancer cells. The underlying mechanisms were demonstrated to mainly involve apoptosis and autophagy, which are related to the oxidative stress-activated AKT/mTOR cell signaling pathway. In bladder cancer xenograft models, a high dose of BCT-100 $(60 \mathrm{mg} / \mathrm{kg})$ was found to remarkably inhibit tumor growth and prolong median survival.

Due to the dysregulation in cellular metabolism, cancer cells require more nutrients to support their proliferation. Therefore, amino acid deprivation has been considered as a promising approach in cancer therapy. Arginine, asparagine, glutamine, methionine, and serine are important amino acids for the tumor growth of certain auxotrophic cancers [17]. One of the most successful examples is L-asparaginase-induced asparagine depletion used in the treatment of acute leukemia. Similarly, arginine depletion therapy has recently been recognized as another encouraging therapeutic strategy in cancer treatment.

As a targeted metabolic anticancer therapy, the efficiency of arginine deprivation is related to the expression of ASS1 and OTC, the two key enzymes in arginine biosynthesis [22]. Previous studies have reported that ASS1- and/or 


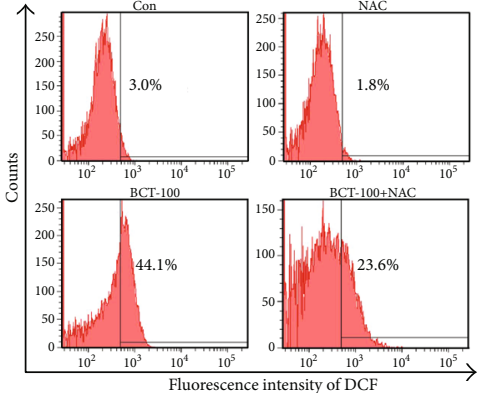

(a)
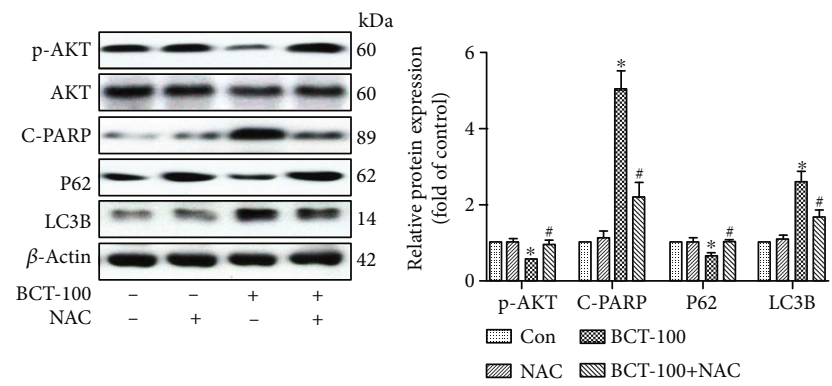

(c)
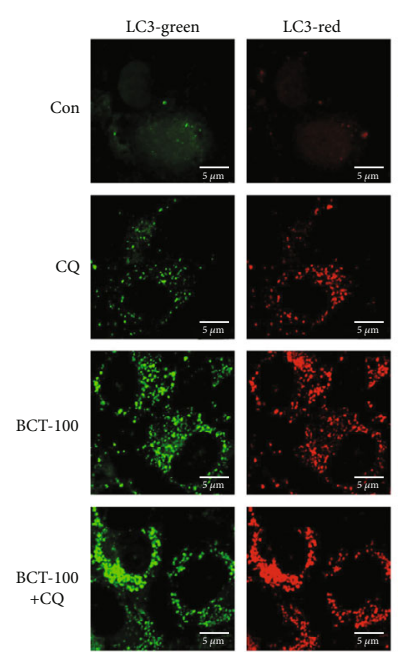

(e)
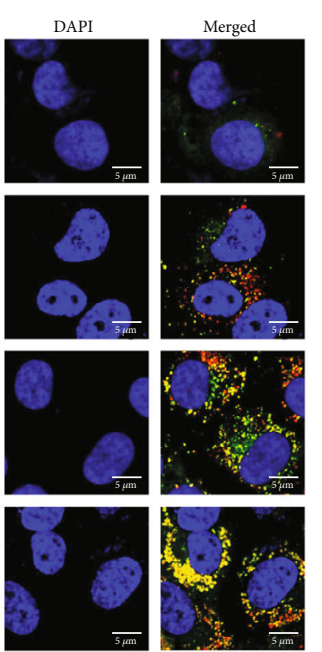
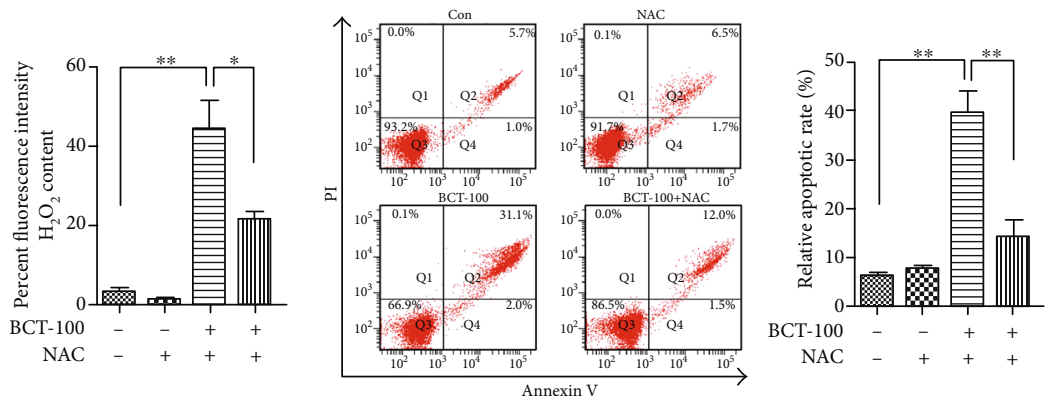

(b)
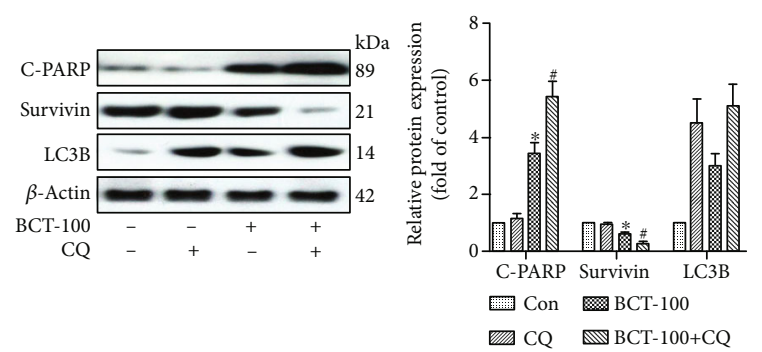

(d)
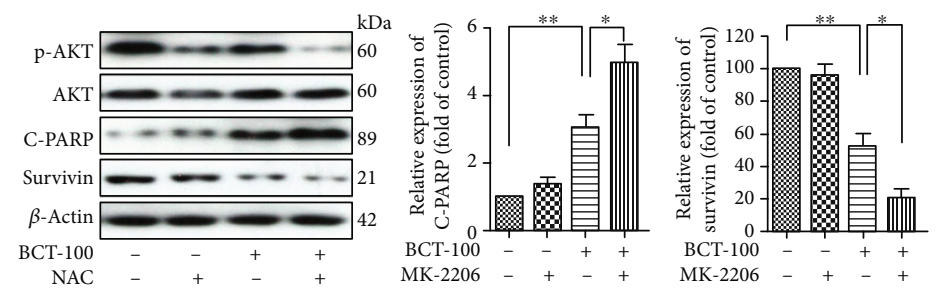

(f)
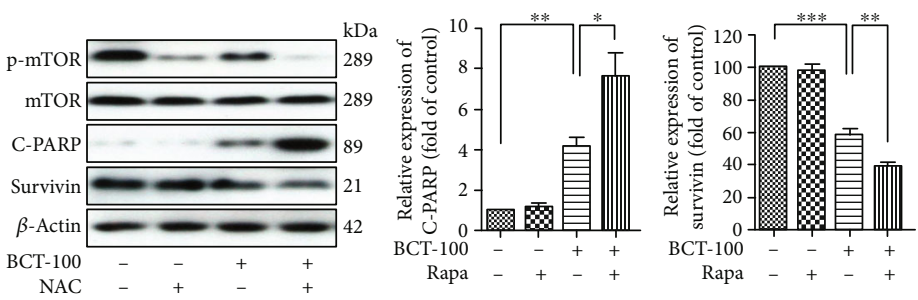

(g)

FIgURE 5: BCT-100-induced ROS initiated cellular apoptosis and autophagy in bladder cancer cells. (a) ROS levels determined by flow cytometry in T24 cells pretreated with NAC $(5 \mathrm{mM}, 1 \mathrm{~h})$ before incubation with BCT-100 $(20 \mathrm{mU} / \mathrm{ml})$ for $24 \mathrm{~h}$ and H2DCFDA ( $1 \mu \mathrm{M})$. (b) Cell apoptotic rate determined by flow cytometry of Annexin V/PI staining in T24 cells pretreated with NAC (5 mM, $1 \mathrm{~h}$ ) before incubation with BCT-100 $(20 \mathrm{mU} / \mathrm{ml})$ for $72 \mathrm{~h}$. (c) Expression of AKT, p-AKT, C-PARP, p62, LC3B, and $\beta$-actin evaluated by Western blot. (d) Expression of C-PARP, Survivin, LC3B, and $\beta$-actin evaluated by Western blot in T24 cells treated with BCT-100 (20 mU/ml) and CQ $(10 \mu \mathrm{M})$ for 3 days. (e) Autophagic flux assessed by confocal microscopy in mRFP-GFP-LC3-transfected T24 cells treated with BCT-100 $(20 \mathrm{mU} / \mathrm{ml})$ and CQ $(10 \mu \mathrm{M})$ for 3 days. (f, g) Expression levels of related proteins tested by Western blot in cells treated with MK-2206 $(2 \mu \mathrm{M})$ and rapamycin $(100 \mathrm{nM})$ with or without BCT-100 for 3 days. $\beta$-Actin was used as a loading control. All data are shown as the mean \pm S.D. of three independent assays. ${ }^{*} p<0.05$ versus control and ${ }^{\sharp} p<0.05$ versus BCT-100, as evaluated by Student's $t$-test. 


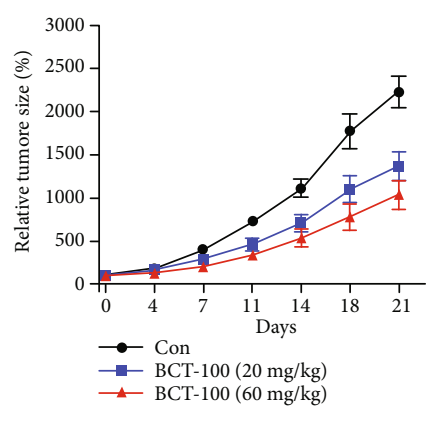

(a)

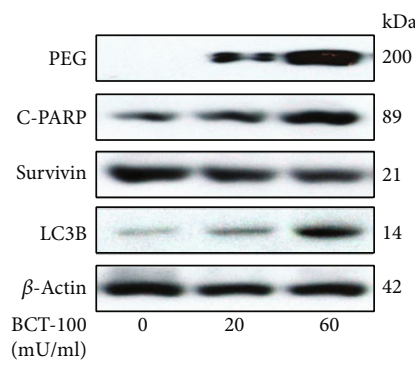

(c)
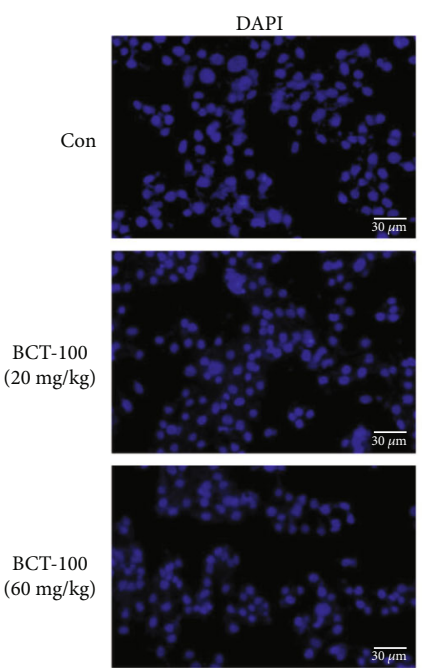

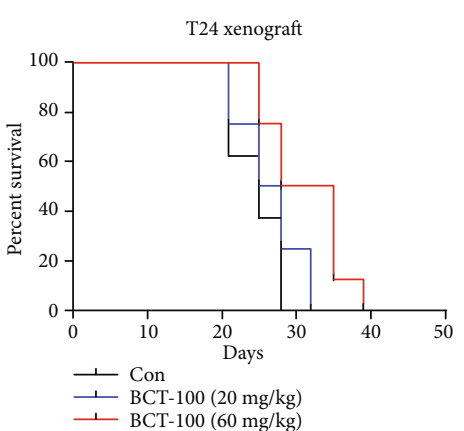

(b)

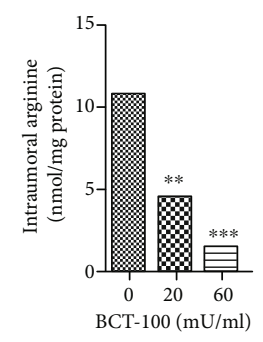

(e)
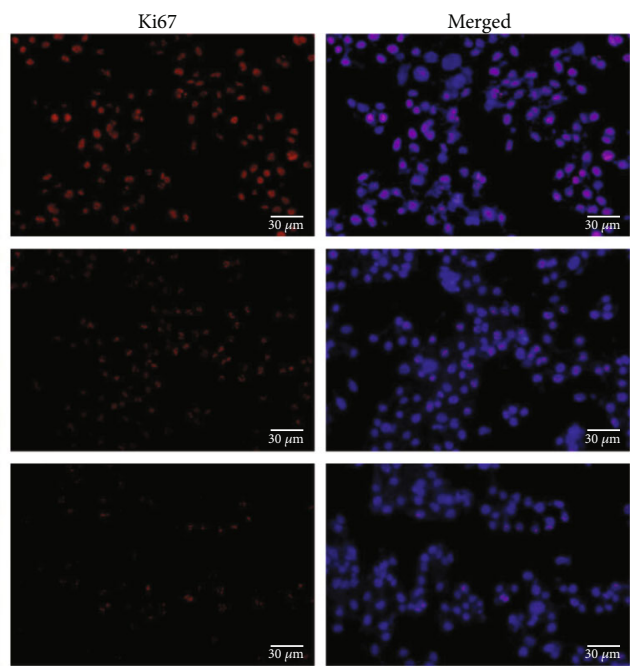

(f)

FIGURE 6: BCT-100 suppressed tumor growth in vivo. (a) Relative tumor volume and (b) median survival of mice in the mock-treated and BCT-100-treated groups $(20 \mathrm{mg} / \mathrm{kg}$ and $60 \mathrm{mg} / \mathrm{kg}$ ) recorded in different time points $(n=7-8$ per group). (c) Expression of PEG, LC3B, C-PARP, and Survivin assessed by Western blot. Serum (d) and intratumoral (e) arginine levels in mice were detected by L-arginine kit. (f) Immunofluorescence staining of Ki67 in mock-treated and BCT-100-treated mice. Bar, $30 \mu \mathrm{m}$. All data are presented as the mean \pm S. D. of three independent experiments. ${ }^{*} p<0.05,{ }^{* *} p<0.01$, and ${ }^{* * *} p<0.001$, as evaluated by Student's $t$-test.

OTC-deficient cell lines are relatively vulnerable to arginine depletors $[13,19]$. For instance, it has been illustrated that ASS1 silencing can enhance the sensitivity of small-cell lung cancer (SCLC) cells to ADI treatment $[18,23]$. Notably, it has been demonstrated that, while knockdown of ASS1 in H69 cells (SCLC) with or without BCT-100 treatment exhibits no significant differences, blocking of OTC sensitizes H841 cells (SCLC) to BCT-100 exposure [13]. Consistently, in our study, we observed that knockdown of ASS1 increases the sensitivity of 5637 cells to BCT-100 treatment (Figures 1(c) and 1(d)). BCT-100 was also observed to display selective anticancer effects on tumor cells, since nontu- morigenic cell line (HaCaT cell), compared with $\mathrm{T} 24$ cell line, was found to be relatively resistant to BCT-100 exposure (Figure S2). Nevertheless, knockdown of OTC was observed to be unable to increase the sensitivity of SCaBER cells to BCT-100 (data not shown). This may be due to the weak basal expression of OTC in SCaBER cells, and there may be other noncanonical pathways that regenerate arginine. However, further studies are needed to support our view.

The underlying mechanisms of arginine depletors might vary in different cancer cell types. ADI, the most wellstudied arginine depletory agent, has been shown to induce mitochondrial dysfunction, ROS production, and autophagy 
in prostate cancer [24]. The Warburg effect was involved after ADI treatment in leiomyosarcoma and melanoma [25]. Akin to ADI in cancer therapy, rhArg has also been demonstrated to exhibit tumor inhibition effects through autophagy induction, oxidative stress, and cell cycle arrest in breast cancer, hepatocellular carcinoma, and acute myeloid leukemia $[19,26,27]$. Therefore, it is necessary to discuss the mechanism of BCT-100 in bladder cancer cells for better clinical outcomes.

Reactive oxygen species (ROS) homeostasis is critical to maintaining the biological functions of cells. Dysregulation of ROS production and metabolism has been shown to cause oxidative stress, which is responsible for several pathological processes, such as autophagy, apoptosis, inflammation, DNA damage, aging, and neurological diseases [28]. The common forms of ROS are the superoxide anion $\left(\mathrm{O}_{2}^{-}\right)$and hydrogen peroxide $\left(\mathrm{H}_{2} \mathrm{O}_{2}\right)$ and their derived forms, hydroxyl radical (HO.) and hydroperoxyl radical (HOO.). Numerous chemotherapeutic drugs have been found to exert antitumor effects via ROS-dependent cytotoxicity in cancer cells in vitro and in vivo [29-31]. In our study, ROS production was boosted and glutathione (GSH) was correspondingly decreased in a dose-dependent fashion after BCT-100 stimulation. Besides, the ROS scavenger NAC could eliminate ROS level thus restore the cell viability. Mitochondrion is an important organelle in various biological activities, including energy generation, signal transduction, cell differentiation, and apoptosis [28]. Since mitochondrion is one of the major sources of ROS, we postulated that BCT-100 can affect mitochondrial function and induce mitochondrial-dependent apoptosis. Consistent with our previous findings, JC-1 staining assay revealed the occurrence of mitochondrial membrane depolarization (MMD) upon BCT-100 treatment. Concurrently, upon mitochondrial stress, Cyt-c along with Smac is released from mitochondria to cytosol, indicating the initiation of mitochondrialmediated apoptosis. Taken together, mitochondrialdependent cytotoxicity due to excessive ROS production is regulated by BCT-100 in bladder cancer cells.

Autophagy is an important cellular event in which cytoplasmic materials are directly degraded by lysosomes [32]. Although cancer cells benefit from autophagy under stressful conditions, such as nutrient deprivation, hypoxia, and oxidative stress, excessive autophagy activities may lead to cell death [33]. Due to the two-faced role of autophagy in cancer therapy as a savior or an executioner, it is necessary to determine the function of autophagy in bladder cancer cells after BCT-100 treatment. In this study, our findings revealed that BCT-100 led to upregulation of Beclin-1 and LC3B, downregulation of p62, and the occurrence of autophagic flux, suggesting autophagy was activated. The autophagy inhibitor CQ was shown to potentiate BCT-100-triggered cytotoxicity, implying that autophagy plays a protective role in BCT-100-treated T24 cells. Consistent with our findings, the prosurvival role of autophagy induced by rhArg has been also demonstrated in laryngeal squamous cell carcinoma [34]. However, autophagy activated by HuArgI (Co)-PEG5000 has been shown to cause autophagic cell death in pancreatic cancer cells [35]. The AKT-mTOR signaling pathway plays an important role in the regulation of apoptosis and autophagy. Activation of AKT-mTOR signaling pathway has been shown to stimulate cell growth. In the present study, BCT100 was shown to inhibit the phosphorylation of AKT and mTOR, without affecting the total protein level, suggesting that the AKT-mTOR signaling pathway is mediated by BCT-100. In addition, NAC was observed to significantly recover the phosphorylation of $\mathrm{AKT}$ and subsequently restore cell viability. Besides, both MK-2206 and rapamycin were shown to enhance the cytotoxicity induced by BCT-100, implying that the AKT-mTOR signaling pathway plays a crucial role in the underlying mechanism.

It is essential to study the in vivo anticancer effects of BCT-100 using xenograft models, which offer a systemic effect with the microenvironment of tumors being present under arginine deprivation. BCT-100 was observed to suppress tumor growth and prolong the survival time with arginine depletion by inducing tumoral apoptosis. The BCT-100 dosage $(20 \mathrm{mg} / \mathrm{kg}$ and $60 \mathrm{mg} / \mathrm{kg}$ ) used in this study was relatively low in comparison with that in other malignant tumor models $[26,36]$, due to the tumor susceptibility to BCT-100 treatment. Furthermore, the body weight of nude mice in medication groups did not markedly decrease contrasted with the control group (data not shown), indicating the side effect of BCT-100 might be acceptable. The most common adverse reactions of cisplatin- and gemcitabine-treated cancer bladder patients include vomiting, nephrotoxicity, and myelosuppression [37, 38]. It may be an option for BCT100 to be combined with chemotherapeutics to diminish the side effects. Since patients receiving BCT-100 in preclinical trials show good tolerance, BCT-100 may be a good alternative therapeutic agent in the clinical management of bladder cancer.

Many clinical trials of rhArg have been initiated in cancer patients, including patients with leukemia, melanoma, HCC, and prostate cancer. The early-phase clinical trial of rhArg has been completed in patients with HCC. It has been shown that the conditions of HCC cancer patients with adequate arginine depletion intervention exhibit significant improvement compared to with those with less arginase treatment (median progression-free survival, 6.4 vs. 1.7 months, 95\% CI: $1.67-1.73, p=0.01$ ) [39]. A study on ADI clinical trials has demonstrated that ADI can prolong progression-free survival in patients with ASS1-deficient mesothelioma (3.2 vs. 2.0 months), and that the overall patient survival in the ADI and control group is 15.7 and 12.3 months, respectively [40]. The side effects of arginine deprivation therapy have been observed to be relatively mild, which include liver dysfunction, neutropenia, fatigue, and nausea $[20,40]$. However, formal phase III clinical trial of ADI on advanced HCC patients has not shown an overall survival benefit in the second-line treatment [41]. Further clinical trials of arginine deprivation in cancer patients are still underway.

Nevertheless, there are several limitations in our study. NAC, CQ, and rapamycin were not employed to confirm the corresponding functions in vivo. It would be more reasonable to use PEG as the normal control in our study, since BCT-100 is a PEG-modified rhArg, although PEG 
is known to be inert to cells [42]. Besides, it is necessary to find out noncanonical pathways to regenerate arginine, which may provide convincing evidence to support our hypothesis.

\section{Conclusions}

In conclusion, we demonstrated that recombinant human arginase (BCT-100) display good anticancer effects against bladder cancer via apoptosis and autophagy regulated by the ROS-activated AKT/mTOR signaling pathway. Our study provides a scientific ground that may facilitate the future clinical development of recombinant human arginase for treating bladder cancer.

\section{Abbreviations}

BCT-100: Bio-Cancer Treatment 100

PEG: $\quad$ Polyethylene glycol

C-PARP: Cleaved poly (ADP-ribose) polymerase

ASS1: Argininosuccinate synthetase

OTC: $\quad$ Ornithine transcarbamylase

ADI: $\quad$ Arginine deiminase

ROS: $\quad$ Reactive oxygen species

Smac: $\quad$ Second mitochondria-derived activator of caspases.

\section{Data Availability}

The data used to support the findings of this study are included within the article.

\section{Conflicts of Interest}

Zhuyun Zhao, Peng Zhang, Wei Li, Dengchuan Wang, Changneng Ke, Yueming Liu, James Chung-Man Ho, and Shi $\mathrm{Xu}$ report no potential conflict of interest. Paul NingMan Cheng is the Chief Executive Officer of Bio-Cancer Treatment International Limited and holds stocks or shares in Bio-Cancer Treatment International Limited.

\section{Authors' Contributions}

Zhuyun Zhao, Peng Zhang, Wei Li, and Dengchuan Wang contributed equally to this work.

\section{Acknowledgments}

This research was supported by the Shenzhen Longhua District Science and Innovation Bureau for Key Laboratory Construction (Grant No. 20160919A0410022), the Medical Institution Foundation of Shenzhen Longhua District (Grant No. 2020038), and the Sanming Project of Medicine in Shenzhen (Grant No. SZSM201801060). The manuscript's preprint is found in the following link: https:// www.researchsquare.com/article/rs-33231/v1.

\section{Supplementary Materials}

Supplementary 1. Supplementary Figure S1: GSH content after BCT-100 treatment.

Supplementary 2. Supplementary Figure S2: the effect of BCT-100 on HaCaT and T24 cell.

\section{References}

[1] F. Bray, J. Ferlay, I. Soerjomataram, R. L. Siegel, L. A. Torre, and A. Jemal, "Global cancer statistics 2018: GLOBOCAN estimates of incidence and mortality worldwide for 36 cancers in 185 countries," CA: A Cancer Journal for Clinicians, vol. 68, no. 6, pp. 394-424, 2018.

[2] M. Foth, N. F. B. Ismail, J. S. C. Kung et al., "FGFR3 mutation increases bladder tumourigenesis by suppressing acute inflammation," The Journal of Pathology, vol. 246, no. 3, pp. 331-343, 2018.

[3] K. J. Kiriluk, S. M. Prasad, A. R. Patel, G. D. Steinberg, and N. D. Smith, "Bladder cancer risk from occupational and environmental exposures," Urologic Oncology, vol. 30, no. 2, pp. 199-211, 2012.

[4] M. Burger, J. W. Catto, G. Dalbagni et al., "Epidemiology and risk factors of urothelial bladder cancer," European Urology, vol. 63, no. 2, pp. 234-241, 2013.

[5] Y. Wang, S. Zou, Z. Zhao, P. Liu, C. Ke, and S. Xu, "New insights into small-cell lung cancer development and therapy," Cell Biology International, vol. 44, no. 8, pp. 1564-1576, 2020.

[6] S. Antoni, J. Ferlay, I. Soerjomataram, A. Znaor, A. Jemal, and F. Bray, "Bladder cancer incidence and mortality: a global overview and recent trends," European Urology, vol. 71, no. 1, pp. 96-108, 2017.

[7] A. K. Schneider, M. F. Chevalier, and L. Derre, "The multifaceted immune regulation of bladder cancer," Nature Reviews Urology, vol. 16, no. 10, pp. 613-630, 2019.

[8] H. Katz, E. Wassie, and M. Alsharedi, "Checkpoint inhibitors: the new treatment paradigm for urothelial bladder cancer," Medical Oncology, vol. 34, no. 10, p. 170, 2017.

[9] L. Fultang, A. Vardon, C. de Santo, and F. Mussai, "Molecular basis and current strategies of therapeutic arginine depletion for cancer," International Journal of Cancer, vol. 139, no. 3, pp. 501-509, 2016.

[10] G. Wu, F. W. Bazer, T. A. Davis et al., "Arginine metabolism and nutrition in growth, health and disease," Amino Acids, vol. 37, no. 1, pp. 153-168, 2009.

[11] R. J. DeBerardinis, J. J. Lum, G. Hatzivassiliou, and C. B. Thompson, "The biology of cancer: metabolic reprogramming fuels cell growth and proliferation," Cell Metabolism, vol. 7, no. 1, pp. 11-20, 2008.

[12] C. de Santo, S. Booth, A. Vardon et al., "The arginine metabolome in acute lymphoblastic leukemia can be targeted by the pegylated-recombinant arginase I BCT-100," International Journal of Cancer, vol. 142, no. 7, pp. 1490-1502, 2018.

[13] S. Xu, S. K. Lam, P. N. Cheng, and J. C. M. Ho, "Recombinant human arginase induces apoptosis through oxidative stress and cell cycle arrest in small cell lung cancer," Cancer Science, vol. 109, no. 11, pp. 3471-3482, 2018.

[14] P. N. Cheng, T. L. Lam, W. M. Lam et al., "Pegylated recombinant human arginase (rhArg-peg5,000mw) inhibits theIn vitroandIn vivoProliferation of human hepatocellular 
carcinoma through arginine depletion," Cancer Research, vol. 67, no. 1, pp. 309-317, 2007.

[15] R. H. Kim, J. M. Coates, T. L. Bowles et al., “Arginine deiminase as a novel therapy for prostate cancer induces autophagy and caspase-independent apoptosis," Cancer Research, vol. 69, no. 2, pp. 700-708, 2009.

[16] P. A. Ott, R. D. Carvajal, N. Pandit-Taskar et al., "Phase I/II study of pegylated arginine deiminase (ADI-PEG 20) in patients with advanced melanoma," Investigational New Drugs, vol. 31, no. 2, pp. 425-434, 2013.

[17] S. Zou, X. Wang, P. Liu, C. Ke, and S. Xu, “Arginine metabolism and deprivation in cancer therapy," Biomedicine \& Pharmacotherapy, vol. 118, article 109210, 2019.

[18] M. P. Kelly, A. A. Jungbluth, B. W. Wu, J. Bomalaski, L. J. Old, and G. Ritter, "Arginine deiminase PEG20 inhibits growth of small cell lung cancers lacking expression of argininosuccinate synthetase," British Journal of Cancer, vol. 106, no. 2, pp. 324332, 2012.

[19] Z. Wang, X. Shi, Y. Li et al., "Blocking autophagy enhanced cytotoxicity induced by recombinant human arginase in triple-negative breast cancer cells," Cell Death \& Disease, vol. 5, no. 12, article e1563, 2014.

[20] T. Yau, P. N. Cheng, P. Chan et al., "Preliminary efficacy, safety, pharmacokinetics, pharmacodynamics and quality of life study of pegylated recombinant human arginase 1 in patients with advanced hepatocellular carcinoma," Investigational New Drugs, vol. 33, no. 2, pp. 496-504, 2015.

[21] A. A. Epenetos, C. Kousparou, and A. Filipovic, "Generation of a selectively cytotoxic fusion protein against p53 mutated cancers," Cancer Research, vol. 76, 2016.

[22] T. L. Lam, G. K. Wong, H. C. Chong et al., "Recombinant human arginase inhibits proliferation of human hepatocellular carcinoma by inducing cell cycle arrest," Cancer Letters, vol. 277, no. 1, pp. 91-100, 2009.

[23] F. Qiu, J. Huang, and M. Sui, “Targeting arginine metabolism pathway to treat arginine-dependent cancers," Cancer Letters, vol. 364, no. 1, pp. 1-7, 2015.

[24] C. A. Changou, Y. R. Chen, L. Xing et al., "Arginine starvationassociated atypical cellular death involves mitochondrial dysfunction, nuclear DNA leakage, and chromatin autophagy," Proceedings of the National Academy of Sciences of the United States of America, vol. 111, no. 39, pp. 14147-14152, 2014.

[25] J. C. Kremer, B. C. Prudner, S. E. S. Lange et al., "Arginine deprivation inhibits the Warburg effect and upregulates glutamine anaplerosis and serine biosynthesis in ASS1-deficient cancers," Cell Reports, vol. 18, no. 4, pp. 991-1004, 2017.

[26] A. K. M. Chow, L. Ng, H. Sing Li et al., "Anti-tumor efficacy of a recombinant human arginase in human hepatocellular carcinoma," Current Cancer Drug Targets, vol. 12, no. 9, pp. 12331243, 2012.

[27] R. Tanios, A. Bekdash, E. Kassab et al., "Human recombinant arginase I(Co)-PEG5000 [HuArgI(Co)-PEG5000]-induced arginine depletion is selectively cytotoxic to human acute myeloid leukemia cells," Leukemia Research, vol. 37, no. 11, pp. 1565-1571, 2013.

[28] T. Ismail, Y. Kim, H. Lee, D. S. Lee, and H. S. Lee, "Interplay between mitochondrial peroxiredoxins and ROS in cancer development and progression," International Journal of Molecular Sciences, vol. 20, no. 18, p. 4407, 2019.

[29] H. J. Shin, H. K. Kwon, J. H. Lee, M. A. Anwar, and S. Choi, "Etoposide induced cytotoxicity mediated by ROS and ERK in human kidney proximal tubule cells," Scientific Reports, vol. 6, no. 1, article 34064, 2016.

[30] M. Wangpaichitr, C. Wu, Y. Y. Li et al., "Exploiting ROS and metabolic differences to kill cisplatin resistant lung cancer," Oncotarget, vol. 8, no. 30, pp. 49275-49292, 2017.

[31] C. Park, H. J. Cha, H. Lee et al., "Induction of G2/M cell cycle arrest and apoptosis by genistein in human bladder cancer T24 cells through inhibition of the ROS-dependent PI3k/Akt signal transduction pathway," Antioxidants, vol. 8, no. 9, p. 327, 2019.

[32] N. M. Kocaturk, Y. Akkoc, C. Kig, O. Bayraktar, D. Gozuacik, and O. Kutlu, "Autophagy as a molecular target for cancer treatment," European Journal of Pharmaceutical Sciences, vol. 134, pp. 116-137, 2019.

[33] J. Liu, L. Fan, H. Wang, and G. Sun, "Autophagy, a doubleedged sword in anti-angiogenesis therapy," Medical Oncology, vol. 33 , no. 1, p. 10, 2016.

[34] C. Lin, Z. Wang, L. Li et al., "The role of autophagy in the cytotoxicity induced by recombinant human arginase in laryngeal squamous cell carcinoma," Applied Microbiology and Biotechnology, vol. 99, no. 20, pp. 8487-8494, 2015.

[35] N. Khalil and R. J. Abi-Habib, "[HuArgI (co)-PEG5000]induced arginine deprivation leads to autophagy dependent cell death in pancreatic cancer cells," Investigational New Drugs, vol. 38, no. 5, pp. 1236-1246, 2020.

[36] T. L. Lam, G. K. Wong, H. Y. Chow et al., "Recombinant human arginase inhibits the in vitro and in vivo proliferation of human melanoma by inducing cell cycle arrest and apoptosis," Pigment Cell \& Melanoma Research, vol. 24, no. 2, pp. 366-376, 2011.

[37] P. Ranganath, L. Einhorn, and C. Albany, "Management of chemotherapy induced nausea and vomiting in patients on multiday cisplatin based combination chemotherapy," BioMed Research International, vol. 2015, 8 pages, 2015.

[38] Y. Cao, Y. He, H. Chen et al., "Phase I study of gemcitabinecisplatin versus pemetrexed cisplatin for patients with advanced or metastatic bladder cancer," Journal of BUON, vol. 23, no. 2, pp. 475-481, 2018.

[39] T. Yau, T. J. Yao, P. Chan, K. Ng, S. T. Fan, and R. T. P. Poon, "A new prognostic score system in patients with advanced hepatocellular carcinoma not amendable to locoregional therapy," Cancer, vol. 113, no. 10, pp. 2742-2751, 2008.

[40] P. W. Szlosarek, J. P. Steele, L. Nolan et al., “Arginine deprivation with pegylated arginine deiminase in patients with argininosuccinate synthetase 1-deficient malignant pleural mesothelioma," JAMA Oncology, vol. 3, no. 1, pp. 58-66, 2017.

[41] G. K. Abou-Alfa, S. Qin, B. Y. Ryoo et al., "Phase III randomized study of second line ADI-PEG 20 plus best supportive care versus placebo plus best supportive care in patients with advanced hepatocellular carcinoma," Annals of Oncology, vol. 29, no. 6, pp. 1402-1408, 2018.

[42] I. A. Ivens, W. Achanzar, A. Baumann et al., "PEGylated biopharmaceuticals," Toxicologic Pathology, vol. 43, no. 7, pp. 959-983, 2015. 